\title{
Étude de la survie et des complications à long terme des prothèses fixes combinées implanto-dento-portées : revue de la littérature
}

\section{RÉSUMÉ}

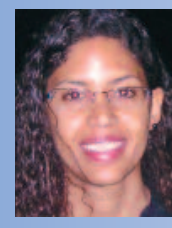

Amandine PARA

DUICP, Paris VII, Chirurgien-dentiste,

72 rue Saint-Blaise,

75020 Paris.
Ancien interne en odontologie,

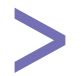
survie des bridges combinés (ou mixtes) implanto-dento-portés et l'incidence des complications biologiques et techniques.

Matériel et méthodes : une recherche effectuée sur Medline, limitée aux dix dernières années de publication, a permis d'identifier des études de cohortes prospectives et rétrospectives ainsi qu'une revue systématique de la littérature sur les bridges combinés. Seules les études ayant un minimum de 5 ans de suivi ont été retenues.

Résultats : les articles retenus ont révélé qu'il n'y a pas de différence significative entre les taux de survie des bridges combinés et celui des bridges implanto-portés conventionnels après 5 ans de fonction. Le taux d'échec des bridges mixtes augmente après une période de suivi de plus de 10 ans et devient significativement plus élevé $(22,2 \%)$ que celui de bridges conventionnels (13,3\%). À 10 ans, le taux de survie implantaire des bridges mixtes semble inférieur $(91,4 \%)$ à celui des piliers implantaires d'un bridge conventionnel (97\%). Selon le type de connexion utilisée, l'incidence des complications biologiques et mécaniques varie : avec une connexion non rigide, l'intrusion dentaire est plus fréquente ( $16 \%$ en moyenne) tandis que la connexion rigide peut entraîner dans certains cas un taux de perte osseuse péri-implantaire annuelle plus élevé (jusqu'à $0,7 \mathrm{~mm} / \mathrm{an}$ au-delà de la 1 re année) qu'une connexion non rigide.

Discussion : la plupart des cas ont été traités avec succès quel que soit le type de connexion (rigide ou non rigide). Dans les cas d'échec
AOS 2011;255:231-242

DOI: $10.1051 /$ aos/2011306

(C) AEOS / EDP Sciences 
rapportés, l'analyse du design de la connexion entre les piliers implantaires et dentaires permet de comprendre la prépondérance d'un type de complication par rapport à l'autre. Faut-il privilégier une connexion rigide plutôt qu'une connexion non rigide ? Davantage d'études cliniques randomisées à long terme seront nécessaires afin de proposer une réponse avec certitude.

Conclusion : les bridges combinés implanto-dento-portés montrent de bons résultats à long terme. Toutefois, les taux de survie globale des implants et des restaurations combinées restent inférieurs à ceux des restaurations uniquement implanto-portées à long terme. Pour l'instant, aucun consensus n'a été obtenu concernant l'indication et la forme idéale de la liaison dent-implant et tous les auteurs préconisent encore une utilisation prudente et rationnelle de cette solution thérapeutique, qui se justifie dans certaines situations cliniques.

\section{Introduction}

$>$

Au cours des deux dernières décennies,

le recours aux implants chez les patients partiellement édentés est devenu une thérapeutique largement admise pour restaurer la forme et la fonction d'une arcade dentaire perturbée. Plusieurs revues systématiques de la littérature et méta-analyses permettent d'établir des taux de survie de plus de $95 \%$ pour les implants et supérieurs à $87 \%$ pour les bridges implanto-portés après 10 ans de fonction [5].

Initialement, les implants introduits par Bränemark étaient recommandés comme piliers uniques de bridges, sans connexion avec des dents naturelles, en raison de la différence de comportement biomécanique entre ces deux entités [5]. La plupart des études longitudinales s'intéressent essentiellement aux bridges uniquement implanto-portés. Cependant, il existe plusieurs situations cliniques d'édentement partiel dans lesquelles une restauration liant une dent et un implant peut être une alternative envisageable.
D'un côté, dans les zones anatomiques à risque ou dans des conditions osseuses insuffisantes par exemple, la conservation de dents peut palier l'impossibilité de placer un nombre suffisant d'implants. D'un autre côté, chez certains patients, la dentition très réduite en raison d'un nombre diminué de dents conservables ou d'un support parodontal affaibli rend difficile la réalisation de prothèses fixées traditionnelles dento-portées. Durant des années, la solution s'est tournée soit vers l'extraction de ces dents et la réalisation d'une prothèse complète amovible muco-portée (ou sur overdenture), soit vers la réalisation d'une " prothèse fixe parodontale " jouant essentiellement le rôle de contention de dents résiduelles mobiles, peu viable à long terme [4].

Sur le plan intellectuel, conserver des dents pouvant éventuellement être liées aux implants apparaît alors comme un concept séduisant. En effet, au-delà d'un retentissement psychologique favorable pour le patient, la conservation 
des dents a pour but de prévenir la résorption osseuse et assure une meilleure transmission des forces aux structures sous-jacentes, tout en maintenant une proprioception.

Sur le plan pratique, connecter des dents et des implants relève cependant du challenge. La dualité de comportement biomécanique de ces deux entités doit être prise en compte : l'attache rigide des implants à l'os crée une réelle ankylose, contrairement au ligament parodontal entourant les dents qui autorise davantage de mobilité. Cette différence de mobilité a conduit certains praticiens à utiliser des dispositifs pour dissocier les contraintes entre la dent et l'implant selon les recommandations de Skalak [1], mais sans vraiment se fonder sur des preuves scientifiques autres que des reports de cas cliniques ou des études rétrospectives à court terme (3 à 5 ans).

On peut rappeler que la liaison dent-implant est apparue dans la littérature au début des années 1980. L'idée de départ pour limiter la mobilité différentielle entre la dent et l'implant a été de réaliser une connexion rigide (bridge monobloc) en intégrant des éléments intramobiles (IME) en matériau viscoélastique dans des implants IMZ [2]. La flexibilité des IME assurait

\section{Matériel et méthodes}

II ne s'agit pas d'une méta-analyse car les données n'ont pas fait l'objet d'une nouvelle analyse statistique, mais d'un état de la question selon les résultats issus d'articles récemment publiés.

\section{Stratégie de recherche}

Toutes les données mentionnées ci-après sont issues de publications recensées sur la base de une rupture de force et l'absorption interne des chocs dans l'implant. Cependant ce dispositif a rapidement été abandonné face aux fréquents cas de fracture répertoriés.

Depuis les IME, le principe d'une dissociation entre les dents et les implants persiste, et deux écoles de pensée s'affrontent : la connexion rigide (bridge monobloc) ou la connexion non rigide (bridge en deux parties reliées par un attachement).

À ce jour, les études restent équivoques sur le comportement et l'efficacité de la liaison dentimplant : certaines indiquent un bon taux de survie implantaire et de survie des bridges avec une connexion rigide et soulignent le risque d'intrusion dentaire lié à une connexion non rigide, tandis que d'autres études récentes préconisent au contraire une connexion non rigide car la connexion rigide dent-implant serait responsable de complications techniques et biologiques telles une perte osseuse péri-implantaire [2].

Le but de la revue de littérature proposée est d'analyser et de comparer les résultats issus de publications récentes à haut niveau de preuve concernant la survie et les complications à long terme des restaurations prothétiques fixées combinant des dents et des implants. données PubMed, avec les mots Mesh « fixed partial dentures " AND « splinting implants » AND " natural tooth " ou " combined tooth implant supported fixed dentures». Les limites " randomized controlled trial, review, clinical trial, meta analysis, multicenter study, case report ", " added to pubmed in the last 10 years", " articles in English, French » ont permis de retenir 10 articles dont 1 revue de littérature, après lecture du 
résumé. Les principales études analysées sont regroupées dans le tableau $\mathrm{I}$.

\section{Études exclues}

En fonction de la durée et de la qualité du suivi des patients (mentionnés dans les matériels et les méthodes des études), certaines publications présélectionnées n'ont pas pu être exploitées. Toutes les études proposant un suivi de moins de 5 ans ont été exclues. De plus, dans les articles retenus, les auteurs ont évalué la survie des restaurations sur des critères cliniques lors de visites de contrôle des patients plutôt que sur des informations écrites consignées dans les dossiers de ces patients.

\begin{tabular}{|c|c|c|c|}
\hline $\begin{array}{l}\text { Lang et al., } \\
2004\end{array}$ & $\begin{array}{l}\text { Revue systématique : } \\
\text { (13 études de cohorte } \\
\text { prospectives/rétrospectives) }\end{array}$ & 5 à 10 ans de suivi & $\begin{array}{l}\text { - Taux de survie des bridges mixtes dent-implant } \\
\text { - Incidence des complications biologiques } \\
\text { et mécaniques }\end{array}$ \\
\hline $\begin{array}{l}\text { Block et al., } \\
2002\end{array}$ & $\begin{array}{l}\text { Étude prospective } \\
\text { comparative }\end{array}$ & $\begin{array}{l}5 \text { ans de suivi } \\
30 \text { patients avec chacun } \\
2 \text { connexions ( } 1 \text { rigide avec } \\
\text { télescope scellé et } 1 \text { non rigide } \\
\text { avec attachement de précision) }\end{array}$ & $\begin{array}{l}\text { Influence du type de connexion } \\
\text { (rigide ou non rigide) sur les implants et les dents : } \\
\text { Critères d'évaluation = } \\
\text { - Perte osseuse } \\
\text { - Profondeur de poche } \\
\text { - Taux de succès cumulé (Albrektsson) } \\
\text { - Taux d'intrusion dentaire } \\
\text { - Importance de la maintenance non planifiée }\end{array}$ \\
\hline $\begin{array}{l}\text { Lin et al., } \\
2006\end{array}$ & $\begin{array}{l}\text { Etude } \\
\text { en éléments finis }\end{array}$ & & $\begin{array}{l}\text { Effet d'un support parodontal } \\
\text { réduit sur la survie des bridges }\end{array}$ \\
\hline $\begin{array}{l}\text { Schlumberger } \\
\text { et al., } 1998\end{array}$ & Revue de littérature & & Analyse des causes d'intrusion dentaire \\
\hline $\begin{array}{l}\text { Naert } \\
\text { et al. } \\
2001\end{array}$ & $\begin{array}{l}\text { Etude clinique } \\
\text { randomisée }\end{array}$ & $\begin{array}{l}N=140 \text { bridges combinés } \\
6,5 \text { ans de suivi moyen } \\
\text { jusqu'15 ans de recul }\end{array}$ & $\begin{array}{l}\text { Analyse radiographique : } \\
\text { comparaison perte osseuse sur implants } \\
\text { combinés versus implants seuls }\end{array}$ \\
\hline
\end{tabular}

\section{Résultats}

Survie globale

des prothèses combinées implanto-dento-portées

La survie des prothèses implanto-dento-portées a été évaluée dans les études comme le main- tien in situ de la prothèse, avec ou sans modifications apparues au cours de la période de suivi. Pour les auteurs, la thérapeutique est considérée comme un échec dès lors que la prothèse $a$ dû être déposée (pour des raisons multiples de complication biologique et/ou mécanique). 
L'analyse des études comparatives sélectionnées ne montre pas de différence significative à court terme entre les taux de survie annuels des bridges combinés (ou mixtes) et les taux de survie des bridges uniquement implanto-portés (>95\%). Cependant, on note au bout de 10 ans de suivi que le taux d'échec des bridges mixtes est significativement plus élevé (22,2\% d'échec soit environ $77 \%$ de survie) que celui des bridges implanto-portés conventionnels (13,3\% d'échec) [5].

En ce qui concerne le taux de survie implantaire, les implants engagés dans un bridge combiné montrent un taux de survie à 10 ans inférieur (91\%) à celui des piliers implantaires d'un bridge conventionnel (97\%). Peu d'études ont évalué le taux de survie des piliers dentaires.

Selon Lang, Pjetursson et al. [5], on note une faible incidence d'échecs liés à une perte des dents support à 5 ans $(3,2 \%)$.

\section{Complications biologiques}

En corollaire au taux de survie à long terme significativement moins élevé pour les bridges combinés, les revues de littérature ont répertorié deux types de complications biologiques principales : une intrusion des dents piliers $(>0,5 \mathrm{~mm})$ et une inflammation gingivale avec perte partielle ou totale de l'ostéointégration des piliers implantaires [9].

Selon les études, l'intrusion dentaire (évaluée par repérage visuel de la jonction entre émail et cément à différents temps) est rapportée en moyenne dans 3 à $5 \%$ des cas à 5 ans. Selon Lang et Pjetursson [5], elle reste moins fréquente avec une connexion rigide (5\% des cas) qu'avec une connexion non rigide (jusqu'à $16 \%$ des cas).

Les autres causes d'échecs de piliers dentaires consécutifs à une complication biologique sem- blent souvent liées à la nécessité de traitement endodontique, une reprise de carie ou une infection. Cependant, les complications des piliers dentaires n'ont pas systématiquement entraîné la dépose du bridge (traitement endodontique réalisé au travers de la couronne par exemple).

En ce qui concerne les complications implantaires, une évaluation radiographique de la perte osseuse péri-implantaire (considérée selon les critères d'Albrektsson) réalisée par Naert [8] atteste que les changements importants du niveau osseux interviennent dans les six premiers mois après mise en place de la prothèse combinée. Ils dépendent du type de connexion employée : rigide ou non rigide. On ne note pas de différence significative de perte osseuse entre le groupe contrôle (uniquement implanto-porté) et le groupe bridge à connexion non rigide. En revanche, la perte osseuse péri-implantaire annuelle semble, selon les résultats de Naert, significativement plus élevée avec une connexion rigide (3\% de perte osseuse en plus soit $0,7 \mathrm{~mm} / \mathrm{an}$ en moyenne à 15 ans) par rapport à un bridge classique ou à un bridge combiné avec une connexion non rigide. Ce résultat n'a cependant pas été relayé par la majorité des auteurs.

\section{Complications techniques}

Toutes les études analysées s'accordent sur la faible incidence globale des complications techniques proprement dites entraînant une réparation ou la réalisation d'une nouvelle prothèse [9].

Les complications les plus fréquemment rencontrées sont une fracture du matériau cosmétique $(9,8 \%$ à 5 ans) puis une perte de rétention du bridge (dévissage du pilier implantaire ou descellement du bridge). Faible à 5 ans, l'incidence des fractures de piliers et de vis aug- 
mente à 10 ans (passant alors de $0,7 \%$ à $24,6 \%$ ). Lorsque ces complications interviennent dans les cinq premières années, peu de modifications sont nécessaires après une première réparation [9].

On ne note pas de différence statistiquement significative entre les complications des bridges à connexion rigide en 1 partie et les complica-

\section{Discussion}

Cette revue de littérature regroupe des études rétrospectives et prospectives sur 5 à 15 ans ainsi qu'une revue systématique. Les études ne peuvent pas être comparées entre elles en raison des différences dans le mode de recherche, le type de population, les sites observés, les critères d'évaluation différents et le délai de suivi. Cependant un certain nombre d'entre elles ont recensé des résultats similaires.

Aucun consensus sur la connexion idéale n'a pour autant été trouvé. En effet une connexion rigide serait néfaste aux implants (perte d'ostéointégration, quelques cas de fracture) et une connexion non rigide serait davantage responsable d'intrusion dentaire.

\section{Influence du type de connexion} sur la survie des bridges combinés à long terme

\section{> Connexion non rigide}

D'un point de vue mécanique, une connexion non rigide (le segment implanto-porté est lié au segment dento-porté par un attachement) autorise un certain degré de liberté du ligament parodontal et des mouvements indépendants de la dent lorsque les charges sont dirigées uniquement sur elle. Les valeurs tions des bridges à connexion rigide en 2 parties (télescopes).

Enfin, on peut signaler qu'il existe une différence significative entre les dents traitées endodontiquement et les dents non traitées, quel que soit le type de connexion (rigide ou non rigide). La fracture intervient souvent au niveau du tenon intracanalaire [1].

intrinsèques de mobilité différentielle entre dents et implants restent certes semblables quel que soit le type de connexion, mais les contraintes transmises à l'os sont moins importantes avec une connexion non rigide. L'analyse réalisée in vitro par Nishimura et al. [7] confirme cet avantage de la connexion non rigide, mais précise que la différence entre connexion rigide et non rigide pour la répartition des charges est faible. De plus, la portion implantaire (souvent transvissée) étant dissociée de la portion dentaire, ce type de connexion permet également la réintervention du praticien en cas de complication mécanique ou biologique sur la partie implantaire. D'un autre côté, la connexion non rigide s'accompagne dans plusieurs cas à long terme d'une intrusion des piliers dentaires, significativement plus fréquente que la connexion rigide.

Chee et al. [2] ont précisé que l'efficacité du système non rigide dépend de la localisation de la jonction, comme le montrent les quatre situations reproduites ci-après.

- Situation 1 (fig. 1a) : connexion non rigide localisée entre le pontique et la dent. La dent supporte seule l'énergie de répartition des contraintes à travers le bridge et risque de réa- 
liser une intrusion à long terme. Le cantilever sur l'implant lui impose une contrainte latérale et augmente le risque de fracture ou de perte osseuse péri-implantaire. Cette connexion, bien que non rigide, semble finalement présenter les mêmes inconvénients qu'une connexion rigide.

- Situation 2 (fig. 1b) : connexion non rigide localisée entre le pontique et l'implant. L'ensemble dent-pontique est indépendant de la portion implantaire et les forces occlusales qui s'appliquent sur la dent sont partagées. Le cantilever est mieux supporté par la dent grâce à la résilience de son ligament desmodontal. Cette configuration paraît acceptable pour diminuer le risque de contrainte latérale et de résorption osseuse péri-implantaire.

- Situation 3 (fig. 1c) : connexion non rigide localisée entre l'implant et le pontique sous forme d'une cuillère à la place d'un attachement classique. Ce système rupteur de force autorise une plus grande liberté de mouvements dans les trois sens de l'espace et réduit I'usure mécanique liée aux frottements verticaux entre partie mâle et partie femelle.

- Situation 4 : structure non rigide modifiée (fig. 2 a à c). Une coiffe dento-portée scellée est recouverte d'une suprastructure en deux parties : une portion dentaire télescope vissée lingualement dans la coiffe et une portion implantaire transvissée. Le segment implantaire

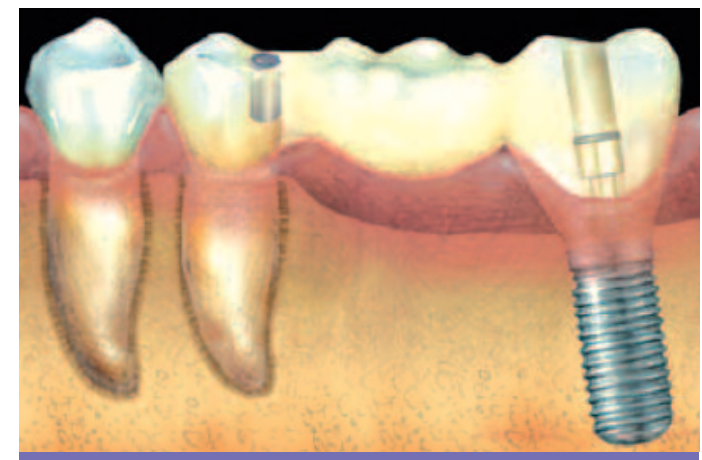

Fig. 1 a Situation $1[2]$
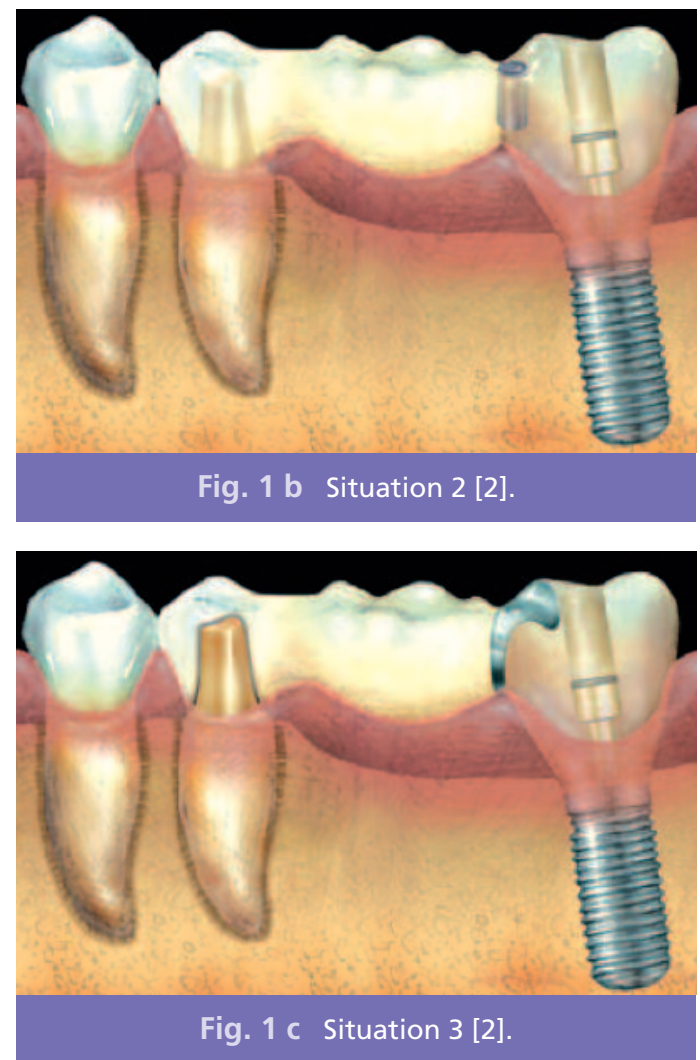
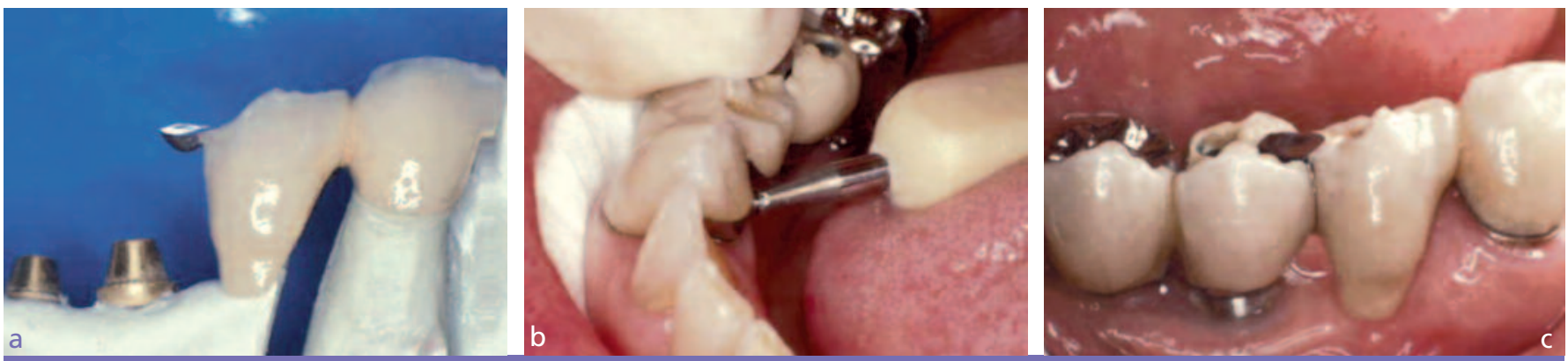

Fig. 2 a à c Connexion non rigide modifiée : portion dento-portée non scellée, vissée lingualement [2]. 
est indépendant, le segment dentaire vient $s^{\prime} y$ appuyer grâce à un attachement en forme de cuillère.

Tout cet accastillage limiterait le risque d'intrusion car la dent est vissée à sa couronne qui bute en sustentation sur la couronne implantaire. L'avantage du système modifié est qu'il permet également le retrait de la partie dentoportée vissée, en plus de la partie implanto-portée. Cette construction permet une réintervention sur chaque segment. Cependant elle occasionne des coûts plus élevés et nécessite une bonne coordination entre le laboratoire de prothèse et le cabinet.

\section{> Connexion non rigide}

Les partisans d'une connexion rigide (bridge monobloc ou télescopé sur une coiffe dentoportée) acceptent la mobilité différentielle entre un implant et une dent naturelle (fig. 3). On sait que, sous une force latérale de $0,1 \mathrm{~N}$, la laxité du ligament parodontal autorise un déplacement physiologique de la couronne dentaire de 50 à $200 \mu \mathrm{m}$ alors que la couronne implantaire se déplace seulement de $4 \mu \mathrm{m}$ reflétant l'ankylose os-implant [8].

Selon cette deuxième école de pensée, la différence de déplacement lors des micromouvements entre les piliers dentaires et implantaires contenus de façon rigide n'aurait pas d'influence néfaste sur la survie du bridge [8]. Ces auteurs suggèrent qu'il y a suffisamment de flexibilité dans la construction implantaire (l'implant et son pilier) pour partager et amortir les charges occlusales. Pourtant, la conséquence d'une connexion rigide qui ressort dans la littérature est un plus fort taux de perte osseuse péri-implantaire avec le risque à long terme de compromettre la survie implantaire.

Toutefois, Nishimura [7] a montré in vitro que, dans un modèle rigide, les contraintes transmises aux implants et à l'os sont supérieures par rapport à un modèle non rigide. Mais l'augmentation des moments des forces subies par les implants au niveau apical et crestal reste relativement faible. Les modèles rigides intégrant deux piliers implantaires assurent une meilleure répartition des forces que les modèles à un seul implant. De même, en augmentant le nombre de piliers dentaires adjacents, la mobilité globale du secteur denté du bridge diminue. Son analyse photo-élastique ne contreindique pas la mise en œuvre d'une connexion rigide : il conclut que les deux types de connexion assurent une répartition correcte des charges. Selon lui, l'intrusion dentaire due à la connexion non rigide pourrait même entraîner une situation de surcharge sur la portion implantaire (qui sera alors la seule partie en occlusion après intrusion du segment dentoporté), plus néfaste que les contraintes transmises par une connexion rigide.
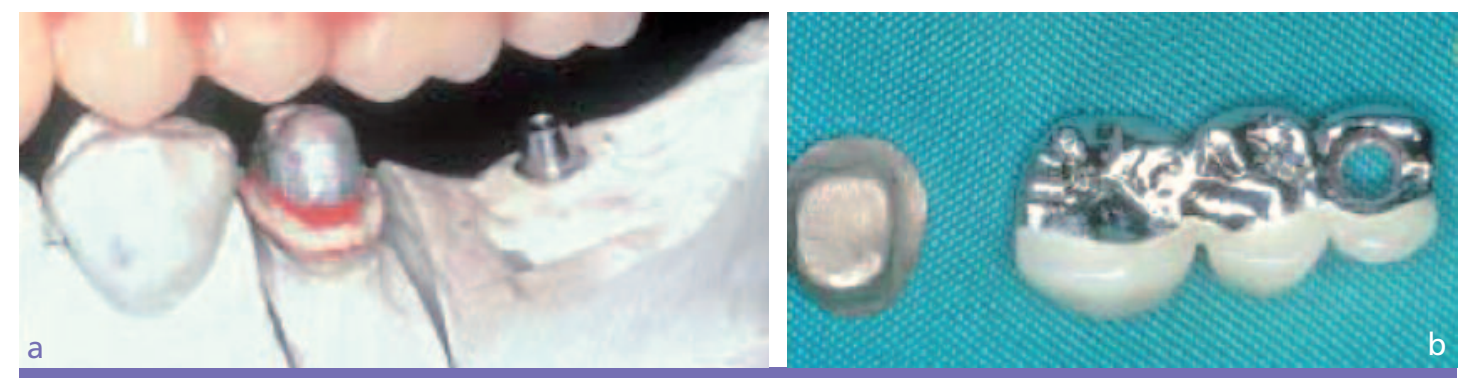

Fig. 3 a et b Connexion rigide. Coiffe en or et bridge télescope, transvissé [1]. 


\section{Intrusion}

Les causes de l'intrusion dentaire restent inconnues. L'étiologie semble être multifactorielle et différente selon les situations [8]. Sous un bridge à connexion rigide, la dent est intruse seule (fig. 4 b). Le hiatus créé représente une voie de pénétration des fluides buccaux et augmente le risque de carie et d'infection. Sous un bridge à connexion non rigide, la dent est intruse avec l'ensemble du segment dentaire dissocié du segment implantoporté (fig. 4 a). Cette intrusion est-elle liée :

- au transfert des charges occlusales qui contraignent la dent dans son alvéole agissant comme des forces orthodontiques?

- à une activation d'ostéoclastes stimulés par une forte contrainte emmagasinée dans la structure?

- à une déficience biomécanique du système prothétique : manque de passivité de l'armature du bridge ?

Pour la connexion non rigide, Chee et al. [2] expliquent l'intrusion dentaire plus fréquente par le frottement entre les parties mâles et femelles de l'attachement. À cet endroit, les forces occlusales dépriment la dent et entraînent une intrusion. Certains auteurs ont même pro- posé l'utilisation d'un lubrifiant sous la prothèse pour diminuer les forces de frottement.

D'autres expliquent l'intrusion par les micromouvements continus de broyage des aliments lors de la mastication, au niveau de la partie femelle, ce qui empêcherait la dent de remonter à son niveau initial (avant une nouvelle dépression du ligament au broyage suivant). La flexibilité de la mandibule a aussi été évoquée pour expliquer le phénomène, mais l'intrusion a été remarquée à la fois au maxillaire et à la mandibule [2].

Pour la connexion rigide, le taux d'intrusion dentaire est significativement plus faible et les explications semblent moins évidentes. L'utilisation d'un ciment de scellement définitif sur le pilier dentaire pourrait limiter le risque d'intrusion. Une alternative consiste à réaliser un bridge à deux étages : une coiffe télescope scellée définitivement sur la dent, sur laquelle la suprastructure est scellée provisoirement. L'avantage est que, si la dent s'intruse, elle reste protégée des percolations par la coiffe, et la suprastructure peut également être retirée en cas de réintervention nécessaire sur la dent ou l'implant.

Quelle que soit la cause réelle, le taux d'intrusion dentaire reste néanmoins faible ( $d$ 'autant plus si la connexion est rigide, environ $3 \%$ )
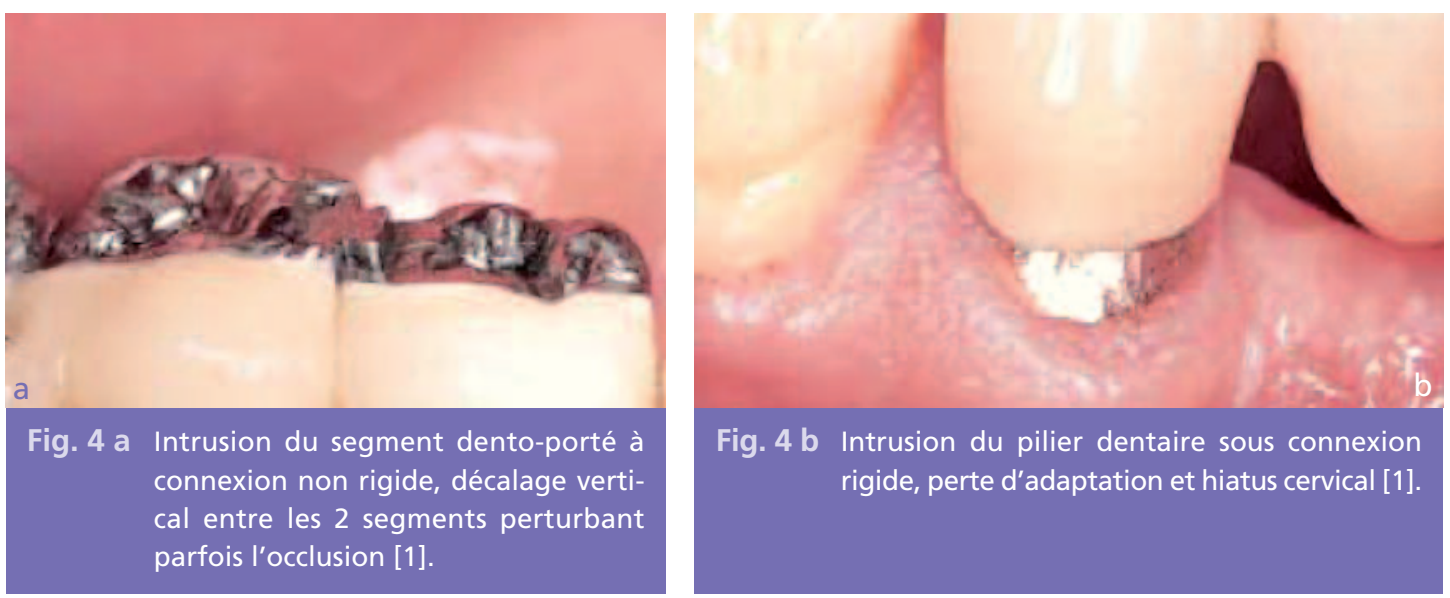
dans toutes les études et la moitié des cas d'intrusion ont été remarqués chez des patients bruxomanes. La sélection initiale des patients ne présentant pas de parafonction permettrait certainement de limiter le risque.

Lorsque I'on considère simultanément les facteurs intrusion et perte osseuse péri-implantaire, aucun consensus sur la connexion idéale n'a été trouvé. Seule l'analyse de chacun des risques possibles (en fonction de la santé parodontale, du nombre de piliers disponibles et de leur localisation) aidera au choix d'un design approprié de la connexion.

\section{Recommandations}

Le nombre d'études avec un haut niveau de preuve étant encore limité, les différents résultats ne permettent pas encore de recommander les bridges combinés, mais fournissent un regard préliminaire sur les complications susceptibles de se produire à long terme et orientent sur la maintenance clinique particulière à prévoir.

Les auteurs gardent des propos modérés : l'incidence réelle et la probabilité de survenue des complications implantaires restent faibles. Actuellement, on ne peut pas garantir une meilleure fiabilité d'un type de connexion par rapport à l'autre.

Une situation particulière doit être signalée. Dans les cas de parodonte réduit, la mobilité des dents naturelles peut augmenter, compliquant alors le comportement biomécanique de la prothèse soumise à des charges occlusales. La liaison rigide des dents ayant un parodonte affaibli sous un bridge traditionnel dento-porté réalise une contention (« prothèse parodontale ») qui diminue efficacement la mobilité du système (diminution du déplacement et de la concentration des contraintes).
Une étude en éléments finis [6] a analysé l'effet de différents niveaux de support parodontal des dents combinées à des implants au sein d'une même prothèse. Ce type de modélisation reste très controversé mais il simule de façon qualitative la complexité de la situation et donne une tendance du comportement biomécanique de la liaison dent-implant sur un support parodontal réduit. Les résultats confirment que les variations du facteur parodontal influencent significativement les forces transmises à l'os sous-jacent et aux implants. La contention rigide de plusieurs dents diminue le stress transmis au parodonte réduit. Lorsque le nombre de dents incluses dans la prothèse est important, les contraintes transmises sont moins élevées : une dent supplémentaire diminue de $4 \%$ le stress sur les implants, et diminue de $47 \%$ les contraintes transmises à l'os. En cas de support parodontal réduit, il est préférable que le bridge combiné intègre une dent supplémentaire afin de répondre aux fonctions physiologiques de façon plus efficace [6].

Ainsi dans un contexte de liaison dent-implant sur un support parodontal réduit, la connexion non rigide doit être utilisée avec précaution : elle stoppe le transfert et la répartition homogène des contraintes entre tous les segments (dentaires et implantaires), et augmente considérablement la concentration du stress sur les implants.

Un groupe d'auteurs [3], réunis lors d'un workshop en 2008, a proposé des « recommandations » sur trois questions :

- recommandation de grade $A$ : dans le secteur postérieur mandibulaire (à partir de la première prémolaire), en cas d'impossibilité de placer un second implant pour des raisons de risque anatomique, un bridge combiné dentimplant est envisageable ; 
- recommandation de grade $\mathrm{B}$ : dans les autres secteurs maxillaire et mandibulaire, pour les mêmes raisons, une dent résiduelle peut être conservée et liée à un implant ;
- avis unanime des auteurs : les bridges combinés sont à envisager avec prudence en pratique prothétique de routine.

\section{Conclusion}

Le pronostic des bridges combinés semble satisfaisant à long terme, quel que soit le type de connexion (rigide ou non rigide).

L'analyse des publications nous incite néanmoins à éviter dès que possible de connecter des dents et des implants, tant que les résultats à long terme ne seront pas étayés par davantage de publications.

Trois paramètres seront à considérer pour décider du design prothétique le plus favorable à un plan de traitement pérenne à long terme :

- la stabilité parodontale,

- le nombre et la localisation des piliers dentaires et implantaires disponibles
- l'absence de parafonction.

Dans l'éventualité d'une telle thérapeutique, mise en œuvre chez un patient non bruxomane, le rapport le plus intéressant du bénéfice de la restauration par rapport au risque d'intrusion ou de surcharge semble s'établir avec une connexion rigide par rapport à une connexion non rigide. Cependant le type de connexion sera décidé en fonction de l'analyse de la situation et du sens clinique du praticien.

Seule une maintenance avertie et régulière facilitera le diagnostic et la gestion anticipée des complications annoncées.

\section{Bibliographie}

1. Block MS, Lirette $D$, Gardiner D, Li L, Finger IM, Hochstedler J, Evans G, Kent JN, Misiek DJ, Mendez AJ, Guerra L, Larsen $\mathrm{H}$, Wood W, Worthington $\mathrm{P}$. Prospective evaluation of implants connected to teeth. Int J Oral Maxillofac Implants 2002 Jul-Aug;17(4):473-87.

2. Chee W, Jivraj S. Connecting implants to teeth. Br Dent J 2006 Nov 25;201(10):629-32.
3. Gotfredsen K, Carlsson GE, Jokstad A, Arvidson Fyrberg $\mathrm{K}$, Berge $M$, Bergendal $B$, Bergendal T, Ellingsen JE, Gunne J, Hofgren M, Holm B, Isidor F, Karlsson $\mathrm{S}$, Klemetti E, Lang NP, Lindh T, Midtbø M, Molin M, Närhi T, Nilner K, Owall B, Pjetursson B, Saxegaard E, Schou S, Stokholm R, Thilander B, Tomasi C, Wennerberg A; Scandinavian Society for Prosthetic Dentistry; Danish Society of Oral Implantology.
Implants and/or teeth: consensus statements and recommendations. J Oral Rehabil 2008 Jan;35 Suppl 1:2-8.

4. Hug S, Mantokoudis D, Mericske-Stern R.

Clinical evaluation of 3 overdenture concepts with tooth roots and implants: 2-year results. Int J Prosthodont 2006;19:236-243.

5. Lang NP, Pjetursson $B E$, Tan K, Brägger U, Egger M, Zwahlen M.

A systematic review 
of the survival and complication rates of fixed partial dentures (FPDs) after an observation period of at least 5 years.

II. Combined tooth-implantsupported FPDs.

Clin Oral Implants Res

2004 Dec;15(6):643-53.

6. Lin $\mathrm{CL}$, Chang $\mathrm{SH}$, Wang JC, Chang WJ.

Mechanical interactions of an implant/tooth-supported system under different periodontal supports and number of splinted teeth with rigid and non-rigid connections. J Dent 2006 Oct;34(9):682-91.

7. Nishimura RD, Ochiai KT,
Caputo AA, Jeong CM. Photoelastic stress analysis of load transfer to implants and natural teeth comparing rigid and semirigid connectors. J Prosthet Dent 1999;81:696-703.

8. Naert IE, Duyck JA, Hosny MM, Quirynen M, van Steenberghe $D$.

Freestanding and tooth-implant connected prostheses in the treatment of partially edentulous patients. Part II: An up to 15 -years radiographic evaluation.

Clin Oral Implants Res 2001;12: 245-251.
9. Nickenig $\mathrm{HJ}$, Spiekermann $\mathrm{H}$, Wichmann M, Andreas SK, Eitner S.

Survival and complication rates of combined tooth-implant-supported fixed and removable partial dentures.

Int J Prosthodont 2008 Mar-Apr;21(2):131-7.

10. Schlumberger $T L$, Bowley JF, Maze GI. Intrusion phenomenon in combination tooth-implant restorations: a review of the literature. J Prosthet Dent 1998 Aug;80(2):199-203.

\section{SUMMARY}

\section{Evaluation of long-term complications}

\section{and survival of the implant-tooth-supported} fixed denture: a literature review

Amandine PARA

$$
\begin{aligned}
& \text { Keywords } \\
& \text { combined tooth- } \\
& \text { implant-supported } \\
& \text { fixed dentures } \\
& \text { - tooth intrusion } \\
& \text { - stiff connection } \\
& \text { non stiff connection }
\end{aligned}
$$

Objectives: The objective of this review is to estimate the survival rate of implanttooth-supported fixed dentures and the incidence of their biological and mechanical complications.

Results: The 5 years (or more) follow up studies and reviews retained on Medline showed no significant difference between the survival rates of combined dentures and the survival rates of conventional implant-supported dentures. The failure rate of combined dentures increases after 10 years and becomes significantly higher $(22,2 \%)$ than failure rate of conventional dentures $(13,3 \%)$. So after 10 years, implants survival of combined dentures seems lower $(91,4 \%)$ than that of a conventional denture (97\%). The incidence of the biological and mechanical complications depends on the type of connection: with a non stiff connection, tooth intrusion is more frequent ( $16 \%$ on average) whereas the stiff connection can increase bone loss.

Conclusion: The combined dentures show good long-term results. However global survival rates of implants involved in combined dentures remain lower than those of implants involved in conventional implant-supported prostheses in the long term. Nowadays, no consensus concerns the indications and the ideal shape of the mixed connection tooth-to-implant. Is it necessary to prefer a stiff connection rather than a not stiff? More clinical studies randomized in the long term will be necessary to assert an answer. All the authors recommend a careful and rational use of this therapeutic, justified in some clinical situations. 